\title{
Random subgraphs in Cartesian powers of regular graphs
}

\author{
Felix Joos \\ Universität Ulm, Germany \\ felix.joos@uni-ulm.de \\ Submitted: Oct 15, 2011; Accepted: Feb 10, 2012; Published: Feb 23, 2012 \\ Mathematics Subject Classification: 05C80
}

\begin{abstract}
Let $G$ be a connected $d$-regular graph with $k$ vertices. We investigate the behaviour of a spanning random subgraph $G_{p}^{n}$ of $G^{n}$, the $n$-th Cartesian power of $G$, which is constructed by deleting each edge independently with probability $1-p$. We prove that $\lim _{n \rightarrow \infty} \mathbb{P}\left[G_{p}^{n}\right.$ is connected $]=e^{-\lambda}$, if $p=p(n)=1-\left(\frac{\lambda_{n}^{1 / n}}{k}\right)^{1 / d}$ and $\lambda_{n} \rightarrow \lambda>0$ as $n \rightarrow \infty$. This extends a result of L. Clark, Random subgraphs of certain graph powers, Int. J. Math. Math. Sci., 32(5):285-292, 2002.
\end{abstract}

\section{Introduction}

For a graph $G$, we denote by $G_{p}$ a random subgraph of $G$ on the same vertex set which includes every edge of $G$ independently of other edges with probability $p \in(0,1)$. Erdős and Rényi [5] were the first to investigate such random graphs. For the complete graph $K_{n}$ with $n$ vertices and $\left(\begin{array}{l}n \\ 2\end{array}\right)$ edges they proved that if $p(n)=\frac{c+\ln n}{n}$ with some $c \in \mathbb{R}$, then $\lim _{n \rightarrow \infty} \mathbb{P}\left[\left(K_{n}\right)_{p}\right.$ is connected] $=\exp \left(-e^{-c}\right)$. Palásti [7] gave an analogous result for the complete bipartite graph $K_{n, n}$ and Ruciński [8] did an improvement for multipartite graphs. The Cartesian power $G^{n}$ of a graph $G$ is the graph with vertex set $V(G)^{n}=V(G) \times \ldots \times V(G)$ where two vertices $\left(x_{1}, \ldots, x_{n}\right)$ and $\left(y_{1}, \ldots, y_{n}\right)$ are adjacent if and only if $x_{i} y_{i} \in E(G)$ for exactly one $i \in\{1, \ldots, n\}$ and $x_{j}=y_{j}$ for all $j \neq i$. Burtin [2] considered a similar problem for the $n$-dimensional 
cube $Q^{n}=\{0,1\}^{n}$. He proved that, if $p>\frac{1}{2}$, then $\lim _{n \rightarrow \infty} \mathbb{P}\left[Q_{p}^{n}\right.$ is connected $]=1$ and if $p<\frac{1}{2}$, then $\lim _{n \rightarrow \infty} \mathbb{P}\left[Q_{p}^{n}\right.$ is connected] $=0$. Erdös and Spencer [6] and Bollobás [1] proved a more precise version of this theorem. They proved that if $p=\frac{1}{2}+\frac{c}{2 n}$ for some $c \in \mathbb{R}$, then $\lim _{n \rightarrow \infty} \mathbb{P}\left[Q_{p}^{n}\right.$ is connected $]=\exp \left(-e^{-c}\right)$.

Clark [3] proved for the complete graph $K_{a}$ with $a$ vertices $(a \geq 2)$ and for the complete bipartite graph $K_{a, a}$ with $a$ vertices in each partition $(a \geq 1)$ a similar result (recall: $\left.K_{2}^{n}=K_{1,1}^{n}=Q^{n}\right)$. In detail he showed, if $1-p=\left(\lambda_{n}^{1 / n} / a\right)^{1 / a-1}$ with $\lambda_{n} \rightarrow$ $\lambda>0$ as $n \rightarrow \infty$, then $\lim _{n \rightarrow \infty} \mathbb{P}\left[\left(K_{a}^{n}\right)_{p}\right.$ is connected $]=e^{-\lambda}$ and similarly for $K_{a, a}$, if $1-p=\left(\lambda_{n}^{1 / n} / 2 a\right)^{1 / a}$ with $\lambda_{n} \rightarrow \lambda>0$ as $n \rightarrow \infty$, then $\lim _{n \rightarrow \infty} \mathbb{P}\left[\left(K_{a, a}^{n}\right)_{p}\right.$ is connected $]=$ $e^{-\lambda}$.

We say that a graph is $d$-regular, if every vertex is adjacent to exactly $d$ vertices. We can generalize the above results to Cartesian powers of arbitrary connected, regular graphs. Keep in mind that the graph $K_{a}$ is $(a-1)$-regular and the graph $K_{a, a}$ is $a$-regular.

Now we state our main result.

Theorem 1.1. Let $G$ be a connected, d-regular graph with $k$ vertices and let $1-p=$ $q=q(n)=\left(\frac{\lambda_{n}^{\frac{1}{n}}}{k}\right)^{1 / d}$, where $\lambda_{n} \rightarrow \lambda>0$ as $n \rightarrow \infty$. Then,

$$
\lim _{n \rightarrow \infty} \mathbb{P}\left[G_{p}^{n} \text { is connected }\right]=e^{-\lambda} \text {. }
$$

Proof: Let $X_{n}$ be the random variable, which denotes the number of isolated vertices in $G_{p}^{n}$. At first we use Lemma 2.2 which is given below

$$
\begin{aligned}
0 & \leq \mathbb{P}\left[G_{p}^{n} \text { disconnected }\right]-\mathbb{P}\left[X_{n}>0\right] \\
& =\mathbb{P}\left[G_{p}^{n} \text { has a component of order } s \text { with } 2 \leq s \leq k^{n} / 2\right]=o(1) \quad(n \rightarrow \infty) .
\end{aligned}
$$

Using Lemma 2.1 which is also given below, we have

$$
\lim _{n \rightarrow \infty} \mathbb{P}\left[G_{p}^{n} \text { disconnected }\right]=\lim _{n \rightarrow \infty} \mathbb{P}\left[X_{n}>0\right]=1-e^{-\lambda}
$$

This completes the proof.

The proof follows the method of Clark [3]. We start with some notations. Let graphs be always simple, finite, and undirected. Let $G$ be a graph. We denote by $V(G)$ the vertex set of $G$ and by $E(G)$ the edge set of $G$. The order $|V(G)|$ of $G$ 
is denoted by $n(G)$ and the size $|E(G)|$ of $G$ is denoted by $m(G)$. For $S \subseteq V(G)$, let $G[S]$ be the subgraph of $G$ induced by $S$ and let $G[S, V(G) \backslash S]$ be the bipartite subgraph of $G$ induced by $S$ and $V(G) \backslash S$. The neighbourhood $N_{G}(v)$ of a vertex $v \in V(G)$ is defined as $\{x \in V(G): x v \in E(G)\}$. The neighbourhood $N_{G}(S)$ of a subset $S \subseteq V(G)$ is defined as $\{x \in V(G) \backslash S: \exists y \in S$ such that $x y \in E(G)\}$. The closed neighbourhood $N_{G}[v]$ of $v$ is $N_{G}(v) \cup\{v\}$ and analogously, $N_{G}[S]=$ $N_{G}(S) \cup S$. The degree $d_{G}(v)$ of $v$ is $\left|N_{G}(v)\right|$ and the maximum degree $\Delta(G)$ of $G$ is $\max \left\{d_{G}(v): v \in V(G)\right\}$. Furthermore, we denote by $d(G)=\frac{2 m(G)}{n(G)}$ the average degree of $G$. We call a graph $d$-regular for $d \in \mathbb{N}$, if every vertex has degree $d$. For arbitrary $v, w \in V(G)$ let $\operatorname{dist}_{G}(v, w)$ be the length of a shortest $v$-w-path. For $S \subseteq V(G)$ and $v \in V(G)$, let $\operatorname{dist}_{G}(S, v)=\min \left\{\operatorname{dist}_{G}(v, w): w \in S\right\}$. The size of the boundary $b_{G}(S)$ of a set $S \subseteq V(G)$ is $|\{v w \in E(G): v \in S, w \in V(G) \backslash S\}|$. Let $b_{G}(s)=\min \left\{b_{G}(S):|S|=s\right\}$. We say that a set $D \subseteq V(G)$ is a dominating set of $G$, if $N_{G}[D]=V(G)$. The domination number $\gamma(G)$ is the minimum order of a dominating set of $G$. To generalize the domination number consider for every $j \in \mathbb{N}$ the $j$-neighbourhood $N_{G}^{j}(v)=\left\{x \in V(G): 0<\operatorname{dist}_{G}(x, v) \leq j\right\}$ and define analogously $N_{G}^{j}(S), N_{G}^{j}[v]$ and $N_{G}^{j}[S]$. Note that $N_{G}^{1}(v)=N_{G}(v)$. We call $D \subseteq V(G)$ a $j$-dominating set of $G$ if $N_{G}^{j}[D]=V(G)$ and let $\gamma^{j}(G)$ be the $j$-domination number of $G$, which is the minimum order of a $j$-dominating set of $G$.

Before we are able to prove Theorem 1.1 we need some lemmas.

Lemma 1.2 (Tillich [9]). Let $G$ be a d-regular graph with $n(G)=k$. Then there exists a $z=z(G)>0$ such that

$$
b_{G^{n}}(s) \geq z d s\left(n-\log _{k} s\right)
$$

for all $n \geq 1$ and for all $1 \leq s \leq k^{n}$.

Lemma 1.3 (Bollobás [1]). Let $G$ be a graph with $\Delta(G) \leq \Delta$ and $\Delta+1<u<$ $n(G)-\Delta-1$. Then there exists a set $U \subseteq V(G)$ with $|U|=u$, such that

$$
\left|N_{G}[U]\right| \geq n(G) \frac{d(G)}{\Delta}\left(1-\exp \left(-\frac{u(\Delta+1)}{n(G)}\right)\right) .
$$

Lemma 1.4. Let $j \in \mathbb{N}$ and $G$ be a graph such that every component has at least $j+1$ elements. Then,

$$
\gamma^{j}(G) \leq \frac{n(G)}{j+1}
$$

The proof of Lemma 1.4 is straightforward and is left to the reader.

the electronic journal of COMbinatorics 19 (2012), \#P47 


\section{Two lemmas for the proof of Theorem 1.1}

Let $X_{n}$ be the random variable, which denotes the number of isolated vertices in $G_{p}^{n}$. Let $\mathbb{E}_{r}[X]=\mathbb{E}[X(X-1) \ldots(X-r+1)]$ the $r$-th factorial moment of a random variable $X$. Note that $\mathbb{E}_{r}\left[X_{n}\right]$ is the expected number of $r$-tuples of different isolated vertices in $G_{p}^{n}$.

Lemma 2.1. Let $G$ be a connected, d-regular graph with $n(G)=k$. Let $q=q(n)=$ $1-p(n)=\left(\frac{\lambda_{n}^{\frac{1}{n}}}{k}\right)^{1 / d}$, with $\lambda_{n} \rightarrow \lambda>0$ as $n \rightarrow \infty$. Then,

$$
\lim _{n \rightarrow \infty} \mathbb{P}\left(X_{n}=0\right)=e^{-\lambda}
$$

Proof: Let $r \in \mathbb{N}$ and $A_{r}=\left\{\left(v_{1}, \ldots, v_{r}\right) \in V\left(G^{n}\right)^{r}: v_{i} \neq v_{j} \forall i, j, i \neq j\right\}$, $B_{r}=\left\{\left(v_{1}, \ldots, v_{r}\right) \in A_{r}: m\left(G^{n}\left[\left\{v_{1}, \ldots, v_{r}\right\}\right]\right) \geq 1\right\}$ and $C_{r}=A_{r} \backslash B_{r}$.

To give an upper bound for $\left|B_{r}\right|$, we choose at first $r-1$ vertices and choose then the last vertex from their neighbourhood. So,

$$
\begin{aligned}
& \left|B_{r}\right| \leq\left(k^{n}\right)_{r-1} \cdot d r n \leq d r n k^{n(r-1)} \quad \text { and } \\
& \left|C_{r}\right|=\left(k^{n}\right)_{r}-\left|B_{r}\right| \geq\left(k^{n}\right)_{r}-d r n k^{n(r-1)}
\end{aligned}
$$

We use $b_{G^{n}}\left(\left\{v_{1}, \ldots, v_{r}\right\}\right) \geq d r(n-r)$ as an estimate for the size of the boundary of one element in $B_{r}$. Now we bound the probability of the event that there exists an $r$-tuple $\left(v_{1}, \ldots, v_{r}\right) \in B_{r}$ or $C_{r}$ containing only isolated vertices in $G_{p}^{n}$. We give bounds for the nonexistence of edges from a vertex of $B_{r}, C_{r}$ to its boundary. We start with $B_{r}$ :

$$
\begin{gathered}
\sum_{\left(v_{1}, \ldots, v_{r}\right) \in B_{r}} \mathbb{P}\left[d_{G_{p}^{n}}\left(v_{1}\right)=\ldots=d_{G_{p}^{n}}\left(v_{r}\right)=0\right] \leq\left|B_{r}\right| q^{d r(n-r)} \\
\leq d r n k^{n(r-1)}\left(\frac{\lambda_{n}^{\frac{1}{n}}}{k}\right)^{r(n-r)}=d r n \frac{\lambda_{n}^{r-\frac{r^{2}}{n}}}{k^{n-r^{2}}}
\end{gathered}
$$

A lower bound for $C_{r}$ :

$$
\begin{aligned}
\sum_{\left(v_{1}, \ldots, v_{r}\right) \in C_{r}} \mathbb{P} & {\left[d_{G_{p}^{n}}\left(v_{1}\right)=\ldots=d_{G_{p}^{n}}\left(v_{r}\right)=0\right]=\left|C_{r}\right| q^{d r n} } \\
& \geq\left(\left(k^{n}\right)_{r}-d r n k^{n(r-1)}\right) \frac{\lambda_{n}^{r}}{k^{n r}}=\lambda_{n}^{r}\left(\frac{\left(k^{n}\right)_{r}}{k^{n r}}-\frac{d r n}{k^{n}}\right)
\end{aligned}
$$


An upper bound for $C_{r}$ :

$$
\sum_{\left(v_{1}, \ldots, v_{r}\right) \in C_{r}} \mathbb{P}\left[d_{G_{p}^{n}}\left(v_{1}\right)=\ldots=d_{G_{p}^{n}}\left(v_{r}\right)=0\right]=\left|C_{r}\right| q^{d r n} \leq k^{n r} \frac{\lambda_{n}^{r}}{k^{n r}}=\lambda_{n}^{r} .
$$

Taking these results together we get

$$
\lambda_{n}^{r}\left(\frac{\left(k^{n}\right)_{r}}{k^{n r}}-\frac{d r n}{k^{n}}\right) \leq \mathbb{E}_{r}\left[X_{n}\right] \leq \lambda_{n}^{r}+d r n \frac{\lambda_{n}^{r-\frac{r^{2}}{n}}}{k^{n-r^{2}}}
$$

Then,

$$
\lim _{n \rightarrow \infty} \mathbb{E}_{r}\left[X_{n}\right]=\lambda^{r}
$$

since $\lim _{n \rightarrow \infty} \frac{\left(k^{n}\right)_{r}}{k^{n r}}=1$. This implies that $X_{n}$ converges to the Poisson distribution with parameter $\lambda$ (see Durrett [4]) and in particular,

$$
\lim _{n \rightarrow \infty} \mathbb{P}\left[X_{n}=0\right]=e^{-\lambda} .
$$

The following lemma is the main step in the proof of Theorem 1.1.

Lemma 2.2. Let $G$ be a connected, d-regular graph with $n(G)=k$ and $1-p=q=$ $\left(\frac{(\ln n)^{\frac{1}{n}}}{k}\right)^{1 / d}$, then

$\mathbb{P}\left[G_{p}^{n}\right.$ has a component of order $s$ with $\left.2 \leq s \leq k^{n} / 2\right]=o(1) \quad(n \rightarrow \infty)$.

In words: With probability approaching 1 as $n \rightarrow \infty$ there is only one component of order greater $\frac{k^{n}}{2}$ and isolated vertices in the random graph $G_{p}^{n}$.

Proof: The following proof is divided into five cases. In each case we give an upper bound for the probability of the event that $G_{p}^{n}$ has a component $S$ of order $2 \leq s \leq k^{n} / 2$. The first two cases use basically that $s$ is small in comparison to $k^{n}$. In the following three cases is $s$ large. In the third case we estimate the probability that there is a component with large $s$ and large boundary. In the remaining two cases the boundary is small and we can use this to complete the proof.

Several inequalities are true only if $n$ is sufficiently large in terms of $G$. In view of the desired statement, we may tacitly assume $n$ to be sufficiently large. Furthermore, 
for the sake of readability we eliminate formally necessary roundings and leave it to the reader to verify that the corresponding inequalities are correct under rounding. Recall the well known inequality $\left(\begin{array}{l}n \\ k\end{array}\right) \leq\left(\frac{e n}{k}\right)^{k}$.

Let $\mathcal{A}_{s}=\left\{S \subseteq V\left(G^{n}\right),|S|=s\right\}$. Now we determine an upper bound for the number of connected subgraphs of order $s$ in $G^{n}$. We pick first one vertex of the connected subgraph. Iteratively we choose every vertex from the neighbourhood of the previous ones:

$$
\mid\left\{S \in \mathcal{A}_{s}: G^{n}[S] \text { is connected }\right\} \mid \leq k^{n} \cdot d n \cdot 2 d n \cdot \ldots \cdot d(s-1) n \leq k^{n} n^{s-1} d^{s-1} s^{s} .
$$

From now we frequently use lemma 1.2. The constant $z=z(G)$ depends only on $G$. It follows that

$$
\begin{aligned}
\sum_{S \in \mathcal{A}_{s}} \mathbb{P}\left[G_{p}^{n}[S] \text { is a component in } G_{p}^{n}\right] & \leq k^{n} n^{s-1} d^{s-1} s^{s} q^{b_{G^{n}}(s)} \\
& \leq \frac{1}{d n} k^{n} n^{s} d^{s} s^{s}\left(\frac{(\ln n)^{\frac{1}{n}}}{k}\right)^{z s\left(n-\log _{k} s\right)} \\
& =\frac{1}{d n} k^{n} n^{s} d^{s} s^{s} \frac{s^{s z}(\ln n)^{s z\left(1-\frac{1}{n} \log _{k} s\right)}}{k^{n s z}} \\
& \leq \frac{1}{d n} \frac{(d n \ln n)^{s} s^{s(z+1)}}{k^{n(s z-1)}}=: \frac{1}{d n} f(s) .
\end{aligned}
$$

Case $1(2 \leq s \leq r, r=r(G, z) \in \mathbb{N}$ sufficiently large in terms of $G)$ : Every vertex in $S \in \mathcal{A}_{s}$ is at most adjacent to $|S|-1$ vertices in $S$. So every vertex is at least adjacent to $d n-(|S|-1)$ vertices in $V\left(G^{n}\right) \backslash S$. Therefore, we have $b_{G^{n}}(s) \geq(d n-(s-1)) s$. Then for all $S \in \mathcal{A}_{s}$ :

$$
\begin{aligned}
\sum_{S \in \mathcal{A}_{s}} \mathbb{P}\left[G_{p}^{n}[S] \text { is a component in } G_{p}^{n}\right] & \leq k^{n} n^{s-1} d^{s-1} s^{s} q^{(d n-(s-1)) s} \\
& \leq k^{n}(d s n)^{s} q^{(d n-r) s} \\
& \leq k^{n}(d s n)^{s} \frac{\ln ^{s} n}{k^{n s-\frac{r s}{d}}} \\
& \leq(d r n)^{r} \ln ^{r} n \frac{1}{k^{n(s-1)-\frac{r s}{d}}}=o(1) \quad(n \rightarrow \infty)
\end{aligned}
$$

Because $r$ depends only on $G$, but not on $n$, we have

$$
\sum_{s=2}^{r} \sum_{S \in \mathcal{A}_{s}} \mathbb{P}\left[G_{p}^{n}[S] \text { is a component in } G_{p}^{n}\right]=o(1) \quad(n \rightarrow \infty) .
$$


Case $2\left(r+1 \leq s \leq \frac{k^{n \frac{z}{z+1}}}{n}=: s_{1}\right)$ : It is an easy exercise to show that $f^{\prime}(s)=$ $f(s) \ln \frac{d n(\ln n) s^{1+z} e^{1+z}}{k^{n z}}$. It follows that $f(s)$ is monotone decreasing in $\left[2, \frac{k^{n} \frac{z}{1+z}}{n}\right]$. If $r$ is large enough, we get

$$
\begin{aligned}
\sum_{s=r+1}^{s_{1}} \sum_{S \in \mathcal{A}_{s}} \mathbb{P}\left[G_{p}^{n}[S]\right. & \text { is a component in } \left.G_{p}^{n}\right] \leq \frac{1}{d n} f(r) \cdot \frac{k^{n \frac{z}{1+z}}}{n} \\
& =\frac{1}{d n} \frac{(d n \ln n)^{r} r^{r(1+z)}}{k^{r n z-n}} \cdot \frac{k^{n \frac{z}{1+z}}}{n} \\
& =\frac{(d n \ln n)^{r} r^{r(1+z)}}{d n^{2}} \frac{1}{k^{n\left(r z-1-\frac{z}{1+z}\right)}}=o(1) \quad(n \rightarrow \infty) .
\end{aligned}
$$

Case $3\left(s_{1} \leq s \leq k^{n} / 2\right)$ : Let $\mathcal{B}_{s}=\left\{S \in \mathcal{A}_{s}: b_{G^{n}}(S) \geq \frac{d s}{j}\left(n-\log _{k} \frac{s}{n^{j^{2}}}\right)\right\}$ and $\mathcal{C}_{s}=\mathcal{A}_{s} \backslash \mathcal{B}_{s}=\left\{S \in \mathcal{A}_{s}: b_{G^{n}}(S)<\frac{d s}{j}\left(n-\log _{k} \frac{s}{n^{j^{2}}}\right)\right\}$ for one $j=j(G, z) \in \mathbb{N} \backslash\{1\}$ large enough in terms of $G$. In this case we only care about $S \in \mathcal{B}_{s}$. The following estimations are easy since we assume that $S$ has a large boundary. Using Lemma 1.4 we get a subset of $S$ which is small compared to $S$ and $(j-1)$-dominates $S$. Keep in mind that for every vertex $v \in S:\left|N_{G^{n}[S]}^{j-1}(v)\right| \leq\left|N_{G^{n}}^{j-1}(v)\right| \leq(d n)^{j}$. We may choose each $S \in \mathcal{B}_{s}$ by first choosing a subset of size $s / j$ which contains the corresponding dominating set (at most $\left(\begin{array}{l}k^{n} \\ s / j\end{array}\right)$ choices) and then picking the remaining $(j-1) s / j$ vertices of $S$ from the neighbours of this set (at most $\left(\begin{array}{c}s / j(d n)^{j} \\ (j-1) s / j\end{array}\right)$ choices). To give an upper bound on the probability that such a set is indeed a component in $G_{p}^{n}$, it is sufficient to demand that none of the edges of the boundary is present in $G_{n}^{p}$.

Since

$$
\begin{aligned}
\sum_{S \in \mathcal{B}_{s}} \mathbb{P}\left[G_{p}^{n}[S]\right. & \text { is a component in } \left.G_{p}^{n}\right] \leq\left(\begin{array}{c}
k^{n} \\
\frac{s}{j}
\end{array}\right)\left(\begin{array}{c}
\frac{s}{j}(d n)^{j} \\
\frac{j-1}{j} s
\end{array}\right) q^{\frac{d s}{j}\left(n-\log _{k} \frac{s}{n^{j^{2}}}\right)} \\
& \leq\left(\frac{j e k^{n}}{s}\right)^{\frac{s}{j}}\left(\frac{e^{j-1}(d n)^{j(j-1)}}{(j-1)^{j-1}}\right)^{\frac{s}{j}}\left(\frac{s(\ln n)^{1-\frac{1}{n} \log _{k} \frac{s}{n^{2}}}}{n^{j^{2}} k^{n}}\right)^{\frac{s}{j}} \leq\left(\frac{O(1) \ln n}{n}\right)^{s},
\end{aligned}
$$

we have

$$
\sum_{s=s_{1}}^{k^{n} / 2} \sum_{S \in \mathcal{B}_{s}} \mathbb{P}\left[G_{p}^{n}[S] \text { is a component in } G_{p}^{n}\right] \leq \sum_{s=s_{1}}^{k^{n} / 2}\left(\frac{O(1) \ln n}{n}\right)^{s}=o(1) \quad(n \rightarrow \infty) .
$$

In the following two cases we only have to look at $S \in \mathcal{C}_{s}$. Because $S \in \mathcal{C}_{s}$ has a small boundary, the probability of the event to have no edge from $S$ to $V(G) \backslash S$ 
increases in comparison to $S \in \mathcal{B}_{s}$. So we need a better upper bound for $\left|\mathcal{C}_{s}\right|$.

Case $4\left(s_{1} \leq s \leq k^{n} / \ln ^{l} n, l=l(G, z, j, c) \in \mathbb{N}\right.$ and $c=c(G, j)$ sufficiently large in terms of $G)$ : Let $H:=G^{n}[S]$. Recall that $d_{G^{n}}(v)=d n$ for every vertex $v \in V(G)$. Then,

$$
\begin{aligned}
d n s & =\sum_{v \in S} d_{G^{n}}(v)=2 m(H)+b_{G^{n}}(S) \\
& <2 m(H)+\frac{d s}{j}\left(n-\log _{k} \frac{s}{n^{j^{2}}}\right)<2 m(H)+\frac{d n s}{j} \\
& \Rightarrow 2 m(H)>d n s\left(1-\frac{1}{j}\right) \\
& \Rightarrow d(H)>d n\left(1-\frac{1}{j}\right)
\end{aligned}
$$

Let $c$ be large enough, $u:=c \frac{s}{n}$ and recall that $\Delta(H) \leq d n$. Then, $d n+1<u<$ $s-d n-1$ for $n$ large enough. Using Lemma 1.3 we get a set $U$ in $H$ such that $|U|=u$ and

$$
\begin{aligned}
\left|N_{H}[U]\right| & \geq s \frac{d n\left(1-\frac{1}{j}\right)}{d n}\left(1-\exp \left(-\frac{u(d n+1)}{s}\right)\right) \\
& =s\left(1-\frac{1}{j}\right)\left(1-\exp \left(-c\left(d+\frac{1}{n}\right)\right)\right) \\
& \geq s\left(1-\frac{2}{j}\right) .
\end{aligned}
$$

Let $t=s\left(1-\frac{2}{j}\right)$. Then $u<t<s$. The set $U$ is adjacent to at least $t-u$ vertices in $H$. Recall that $\left(\begin{array}{c}d n u \\ i\end{array}\right) \leq 2^{d n u}$ for all $0 \leq i \leq d n u$. So, we get an upper bound for $\left|\mathcal{C}_{s}\right|$ by first picking $u$ vertices, then the vertices in their neighbourhood and at the end the remaining vertices, which are at most $2 s / j$ :

$$
\left|\mathcal{C}_{s}\right| \leq\left(\begin{array}{c}
k^{n} \\
u
\end{array}\right) 2^{d n u}\left(\begin{array}{c}
k^{n} \\
\frac{2 s}{j}
\end{array}\right) \leq\left(\frac{e k^{n}}{u}\right)^{u} 2^{d n u}\left(\frac{j e k^{n}}{2 s}\right)^{\frac{2 s}{j}}
$$

Recall that $z=z(G)$ is fixed for a fixed graph $G$ and therefore we have that $z-\frac{c}{n}-\frac{2}{j}>$ 0 , if $j$ is large enough and $n \geq n_{0}(G)$. So we have an upper bound for probability 
that $G_{p}^{n}[S]$ is a component in $G_{p}^{n}$ :

$$
\begin{aligned}
\sum_{S \in \mathcal{C}_{s}} \mathbb{P} & {\left[G_{p}^{n}[S] \text { is a component in } G_{p}^{n}\right] \leq\left|\mathcal{C}_{s}\right| q^{b_{G}(s)} } \\
& \leq\left(\frac{e n k^{n}}{c s}\right)^{\frac{c s}{n}} 2^{d c s}\left(\frac{j e k^{n}}{2 s}\right)^{\frac{2 s}{j}}\left(\frac{(\ln n)^{\frac{1}{n}}}{k}\right)^{s z\left(n-\log _{k} s\right)} \\
& =\left[2^{d c}\left(\frac{e n}{c}\right)^{\frac{c}{n}}\left(\frac{j e}{2}\right)^{\frac{2}{j}}\left(\frac{s}{k^{n}}\right)^{z-\frac{c}{n}-\frac{2}{j}}(\ln n)^{z\left(1-\frac{1}{n} \log _{k} s\right)}\right]^{s} \\
& \leq\left[O(1)(\ln n)^{z\left(1-\frac{1}{n} \log _{k} s\right)-l\left(z-\frac{c}{n}-\frac{2}{j}\right)}\right]^{s} .
\end{aligned}
$$

In (2.3) we use the fact that $\frac{s}{k^{n}}$ is maximal, if $s$ is maximal. Since $z>\frac{c}{n}+\frac{2}{j}$, we can choose $l$ so large that $z\left(1-\frac{1}{n} \log _{k} s\right)-l\left(z-\frac{c}{n}-\frac{2}{j}\right)<-\alpha<0$ for some $\alpha>0$. Hence,

$$
\sum_{S \in \mathcal{C}_{s}} \mathbb{P}\left[G_{p}^{n}[S] \text { is a component in } G_{p}^{n}\right] \leq\left[O(1)(\ln n)^{-\alpha}\right]^{s}
$$

and it follows that

$$
\sum_{s=s_{1}}^{\frac{k^{n}}{\ln ^{l} n}} \sum_{S \in \mathcal{C}_{s}} \mathbb{P}\left[G_{p}^{n}[S] \text { is a component in } G_{p}^{n}\right] \leq \sum_{s=s_{1}}^{\frac{k^{n}}{\ln ^{l} n}}\left[O(1)(\ln n)^{-\alpha}\right]^{s}=o(1) \quad(n \rightarrow \infty) .
$$

Case $5\left(k^{n} / \ln ^{l} n \leq s \leq k^{n} / 2\right)$ : Let again $H:=G^{n}[S]$. Furthermore, consider the set $T=\left\{v \in S: d_{H}(v) \geq d n-\log _{k}^{2} n\right\}$, and define $t:=|T|$ and $H_{1}:=G^{n}[T]=H[T]$. We will show that the degree of every vertex in $H_{1}$ is close to the maximum degree and because of that the graph $H_{1}$ has large average degree. This is done as follows. We start with a partition of the edges of $H$

$$
\begin{aligned}
2 m\left(H_{1}\right) & =2 m(H)-2 m(H[S \backslash T])-2 m(H[S \backslash T, T]) \\
& \geq d s\left(\frac{j-1}{j} n+\frac{1}{j} \log _{k} \frac{s}{n^{j^{2}}}\right)-2 \cdot d n(s-t) \\
& =d s\left(\frac{j-1}{j} n+\frac{1}{j} \log _{k} \frac{s}{n^{j^{2}}}-\frac{2 n}{s}(s-t)\right) .
\end{aligned}
$$

For (2.4) keep the definition of $\mathcal{C}_{s}$ in mind and note that every upper bound for the boundary of $H$ corresponds to a lower bound for $m(H)$. 
Since $s \geq \frac{k^{n}}{\ln ^{l} n}$, we have:

$$
\frac{j-1}{j} n+\frac{1}{j} \log _{k} \frac{s}{n^{j^{2}}} \geq \log _{k} \frac{k^{n}}{n^{j} \ln ^{l / j} n} \geq n-(j+1) \log _{k} n .
$$

Let $\epsilon:=\frac{(j+1) d}{\log _{k} n}$. By the definition of $T$ and $\mathcal{C}_{s}$ it follows that

$$
\begin{gathered}
d s n-(s-t) \log _{k}^{2} n \geq \sum_{v \in S} d_{H}(v) \\
>d s n-\frac{d s}{j}\left(n-\log _{k} \frac{s}{n^{j^{2}}}\right) \geq d s\left(n-(j+1) \log _{k} n\right) .
\end{gathered}
$$

It follows that

$$
t \geq s\left(1-\frac{(j+1) d}{\log _{k} n}\right)=s(1-\epsilon)
$$

Take (2.6) and (2.7) and plug it into (2.5), such that

$$
\begin{aligned}
2 m\left(H_{1}\right) & \geq d s\left(n-(j+1) \log _{k} n-\frac{2 n}{s} s \epsilon\right) \\
& =d s\left((1-2 \epsilon) n-(j+1) \log _{k} n\right) \geq d s n(1-3 \epsilon) .
\end{aligned}
$$

Taking everything together we get a lower bound for $d\left(H_{1}\right)$ :

$$
d\left(H_{1}\right) \geq \frac{2 m\left(H_{1}\right)}{t} \geq \frac{2 m\left(H_{1}\right)}{s} \geq d(1-3 \epsilon) n .
$$

Let $u:=\frac{k^{n}}{\ln ^{3 l} n}$, such that $d n+1<u<t-d n-1$. Using Lemma 1.3 again, we get a set $U \subset S$ mit $|U|=u$, such that

$$
\begin{aligned}
\left|N_{H}[U]\right| \geq\left|N_{H_{1}}[U]\right| & \geq t \frac{d(1-3 \epsilon) n}{d n}\left(1-\exp \left(-\frac{u(d n+1)}{t}\right)\right) \\
& \geq s(1-\epsilon)(1-3 \epsilon)\left(1-\exp \left(-\frac{k^{n}(d n+1)}{t \ln ^{3 l} n}\right)\right) \\
& \geq s(1-\epsilon)^{2}(1-3 \epsilon) \geq s(1-5 \epsilon) .
\end{aligned}
$$

Let $c:=s-5 \epsilon s$, such that $u<c<s, w:=5 \epsilon s$ and define $y:=\log _{k}^{2} n$. Recall the definition of $T$. Every vertex in $H_{1}$ has at most $y$ adjacent vertices which are not in 
$H$. Then

$$
\begin{aligned}
\left|\mathcal{C}_{s}\right| & \leq\left(\begin{array}{c}
k^{n} \\
u
\end{array}\right)\left(\sum_{\left(k_{1}, \ldots, k_{u}\right) \in\{0, \ldots, y\}^{u}} \prod_{i=1}^{u}\left(\begin{array}{c}
d n \\
k_{i}
\end{array}\right)\right)\left(\begin{array}{c}
k^{n} \\
w
\end{array}\right) \\
& \leq\left(\frac{e k^{n}}{u}\right)^{u}(y+1)^{u}\left(\begin{array}{c}
d n \\
y+1
\end{array}\right)^{u}\left(\frac{e k^{n}}{w}\right)^{w} \\
& \leq\left(\frac{e k^{n}}{u}\right)^{u}(y+1)^{u}\left(\frac{d n e}{y+1}\right)^{u(y+1)}\left(\frac{e k^{n}}{w}\right)^{w} \\
& \leq\left(d e^{2} n \ln ^{3 l} n\right)^{u}\left(\frac{d n e}{\log _{k}^{2} n}\right)^{u y}\left(\frac{e k^{n}}{5 \epsilon s}\right)^{5 \epsilon s} .
\end{aligned}
$$

Recall that $l \geq 2$ and $\frac{k^{n}}{\ln ^{l} n} \leq s \leq \frac{k^{n}}{2}$. It follows that

$$
\begin{gathered}
0<\frac{u}{s}<\frac{u y}{s}<\frac{1}{\ln ^{2} n}, \\
z\left(1-\frac{1}{n} \log _{k} s\right)-\frac{2 u y}{s} \leq z \frac{l}{n} \log _{k} \ln n-\frac{4}{\ln ^{2} k \ln ^{3 l-2} n} \leq 0 .
\end{gathered}
$$

For (2.10) keep in mind $s \leq \frac{k^{n}}{2}, z-5 \epsilon>0$ and see (2.8), (2.9) above. Let $\alpha:=2^{-z}<1$ and hence $\frac{1+\alpha}{2}<1$ with $z$ as in Lemma 1.2. Then,

$$
\begin{array}{rl}
\sum_{S \in \mathcal{C}_{s}} & \mathbb{P}\left[G_{p}^{n}[S] \text { is a component in } G_{p}^{n}\right] \leq\left|\mathcal{C}_{s}\right| q^{b_{G}(s)} \\
& \leq\left(d e^{2} n \ln ^{3 l} n\right)^{u}\left(\frac{d n e}{\log _{k}^{2} n}\right)^{u y}\left(\frac{e k^{n}}{5 \epsilon s}\right)^{5 \epsilon s}\left(\frac{(\ln n)^{\frac{1}{n}}}{k}\right)^{s z\left(n-\log _{k} s\right)} \\
& =\left[\left(d e^{2} n \ln ^{3 l} n\right)^{\frac{u}{s}}\left(d n e \ln ^{2} k\right)^{\frac{u y}{s}}\left(\frac{e}{5 \epsilon}\right)^{5 \epsilon}\left(\frac{s}{k^{n}}\right)^{z-5 \epsilon}(\ln n)^{z\left(1-\frac{1}{n} \log _{k} s\right)-\frac{2 u y}{s}}\right]^{s} \\
& \leq\left[\left(d^{2} e^{3} n^{2} \ln ^{3 l} n \ln ^{2} k\right)^{\frac{1}{\ln ^{2} n}}\left(\frac{e}{5 \epsilon}\right)^{5 \epsilon} 2^{5 \epsilon-z}\right]^{s} \\
& \leq\left(\frac{1+\alpha}{2}\right)^{s} .
\end{array}
$$

In $(2.11)$ we use $\left(d^{2} e^{3} n^{2} \ln ^{3 l} n \ln ^{2} k\right)^{\frac{1}{\ln ^{2} n}} \rightarrow 1(n \rightarrow \infty),\left(\frac{e}{5 \epsilon}\right)^{5 \epsilon} \rightarrow 1(\epsilon \rightarrow 0)$ and $\epsilon \rightarrow 0$ 
$(n \rightarrow \infty)$. Taking everything into consideration leads to

$$
\sum_{s=\frac{k^{n}}{\ln ^{n} n}}^{\frac{k^{n}}{2}} \sum_{S \in \mathcal{C}_{s}} \mathbb{P}\left[G_{p}^{n}[S] \text { is a component in } G_{p}^{n}\right] \leq \sum_{s=\frac{k^{n}}{\ln ^{1} n}}^{\frac{k^{n}}{2}}\left(\frac{1+\alpha}{2}\right)^{s}=o(1) \quad(n \rightarrow \infty) .
$$

Remark. Keep in mind that if $\tilde{q} \leq q$, then $\tilde{q}^{b_{G}(s)} \leq q^{b_{G^{n}}(s)}$. Since $\lambda_{n} \rightarrow \lambda>0$ as $n \rightarrow \infty$, there exists a $N \in \mathbb{N}$, such that $\lambda_{n} \leq \ln n$ for all $n \geq N$. Because of that Lemma 2.2 remains correct if we replace $q=\left(\frac{(\ln n)^{\frac{1}{n}}}{k}\right)^{1 / d}$ by $q=\left[\lambda_{n}^{\frac{1}{n}} / k\right]^{1 / d}$.

\section{References}

[1] B. Bollobás. The evolution of the cube. In Combinatorial mathematics (Marseille-Luminy, 1981), volume 75 of North-Holland Math. Stud., pages 9197. North-Holland, Amsterdam, 1983.

[2] J. D. Burtin. The probability of connectedness of a random subgraph of an n-dimensional cube. Problemy Peredači Informacii, 13(2):90-95, 1977.

[3] L. Clark. Random subgraphs of certain graph powers. Int. J. Math. Math. Sci., 32(5):285-292, 2002.

[4] R. Durrett. Probability. The Wadsworth \& Brooks/Cole Statistics/Probability Series. Wadsworth \& Brooks/Cole Advanced Books \& Software, Pacific Grove, CA, 1991. Theory and examples.

[5] P. Erdős and A. Rényi. On random graphs. I. Publ. Math. Debrecen, 6:290-297, 1959.

[6] P. Erdős and J. Spencer. Evolution of the n-cube. Comput. Math. Appl., 5(1):3339, 1979.

[7] I. Palásti. On the connectedness of random graphs. In Studies in Mathematical Statistics: Theory and Applications, pages 105-108. Akad. Kiadó, Budapest, 1968.

[8] A. Ruciński. The $r$-connectedness of $k$-partite random graph. Bull. Acad. Polon. Sci. Sér. Sci. Math., 29(7-8):321-330, 1981.

[9] J.-P. Tillich. Edge isoperimetric inequalities for product graphs. Discrete Math., 213(1-3):291-320, 2000. 